\title{
Witnessing Entanglement
}

\author{
Marisol N. Beck* and M. Beck† \\ *Department of Physics, Harvey Mudd College, 301 Platt Blvd., Claremont, CA 91711 \\ $\dagger$ Department of Physics, Whitman College, 345 Boyer Ave., Walla Walla, WA 99362
}

\begin{abstract}
An entangled state of a two-particle system is a quantum state that cannot be separated-it cannot be written as the product of states of the individual particles. One way to tell if a system is entangled is to use it to violate a Bell inequality (such as the Clauser-Horne-Shimony-Holt, CHSH, inequality), because entanglement is necessary to violate these inequalities. However, there are other, more efficient measurements that determine whether or not a system is entangled; an operator that corresponds to such a measurement is referred to as an entanglement witness. We present the theory of witness operators, and an undergraduate experiment that measures an entanglement witness for the joint polarization state of two photons. We are able to produce states for which the expectation value of the witness operator is entangled by more than 160 standard deviations.
\end{abstract}

Keywords: Quantum Mechanics, Entanglement, Quantum Measurement, Quantum Information. PACS: 03.67.Mn, 03.67.Bg, 42.50.Dv.

\section{INTRODUCTION}

Entanglement is a (perhaps the) feature that distinguishes quantum mechanics from classical mechanics. Entanglement is necessary for a diverse range of uniquely quantum mechanical effects such as quantum cryptography, quantum teleportation and quantum computing. ${ }^{1}$

Mathematically, entangled states are those quantum states that cannot be written as the product of the states of the individual particles. Thus, if $\left|\psi_{\text {ent }}\right\rangle$ represents an entangled state of a bipartite system, and $\left|\psi_{A}\right\rangle$ and $\left|\psi_{B}\right\rangle$ are the states of the individual particles, then

$$
\left|\psi_{\text {ent }}\right\rangle \neq\left|\psi_{A}\right\rangle \otimes\left|\psi_{B}\right\rangle
$$

where $\otimes$ represents the direct product.

In Eq. (1) $\left|\psi_{\text {ent }}\right\rangle$ is an entangled pure state. It has been shown that for every bipartite pure-state, there exists a Bell inequality that is violated, ${ }^{2,3}$ this means that there exists, at least in principle, a method to experimentally detect that entanglement.

However, real experimental systems never exist in pure states. One must assume that the state of an experiment will yield a mixed state that must be described by density operator $\hat{\rho} .^{4}$ A mixed state is separable, and hence not entangled, if it can be written as a weighted sum of product states:

$$
\hat{\rho}_{s e p}=\sum_{i} p_{i} \hat{\rho}_{A i} \otimes \hat{\rho}_{B i},
$$

where the $p_{i}$ 's are nonnegative real numbers, and the normalization condition is that they must sum to 1 .

An observable that is able to detect entanglement is referred to as an entanglement witness. ${ }^{5,6}$ Bell inequalities were the first entanglement witnesses, but there are other, more efficient, observables that are capable of detecting entanglement. For example, the minimum number of measurements needed to determine a Bell inequality for bipartite qubits (two, 2state particles) is four, whereas it is possible to construct an entanglement witness for these same qubits that requires only three measurements. ${ }^{7}$ The reason Bell inequalities require more measurements is because they are capable of ruling out any localrealistic model, whereas other entanglement witnesses assume the validity of quantum mechanics, and merely seek to determine whether or not a particular system is entangled.

Experiments with entangled photons have been previously performed in undergraduate laboratories. ${ }^{4,8-}$ ${ }^{12}$ These experiments include tests of Bell inequalities, which prove that the states used in those experiments were entangled. However, we know of no previous undergraduate experiments that measure the types of entanglement witnesses that we describe here. These witnesses require only three measurements, not four. Furthermore, we demonstrate that our witness operators are able to detect entanglement in situations where the Clauser-Horne-Shimony-Holt, CHSH, inequality, ${ }^{8,9}$ which is the most commonly used Bell inequality, does not.

A full discussion of mixed-state density operators and witness operators is well beyond the scope of this article. For a discussion of density operators that is accessible to undergraduates, see Ref. [4]. For a more 
complete discussion of witness operators, see Refs. [3] and [6].

\section{THEORY}

\section{Schmidt Decomposition}

Before discussing the general problem of identifying entanglement in arbitrary mixed state systems, let's first consider entanglement of pure states. Suppose that system $A$ has dimension $N$ and system $B$ has dimension $M$. An arbitrary pure state of the joint system can be written as

$$
\begin{aligned}
|\psi\rangle & =\sum_{i}^{N} \sum_{j}^{M} c_{i j}\left|\alpha_{i}\right\rangle_{A} \otimes\left|\beta_{j}\right\rangle_{B} \\
& =\sum_{i}^{N} \sum_{j}^{M} c_{i j}\left|\alpha_{i} \beta_{j}\right\rangle .
\end{aligned}
$$

The Schmidt decomposition of $|\psi\rangle$ determines two new sets of basis vectors $\left|a_{i}\right\rangle_{A}$ and $\left|b_{i}\right\rangle_{B}$, such that

$$
|\psi\rangle=\sum_{i}^{R} \lambda_{i}\left|a_{i} b_{i}\right\rangle
$$

The number $R$ is called the Schmidt rank of the system, and $R \leq \min (N, M)$. This is a simplification, because we have gone from a double sum to a single sum. The fact that the Schmidt decomposition of $|\psi\rangle$ exists is proven in Ref. [1].

The Schmidt decomposition is useful for several reasons. The Schmidt rank of any pure product state is 1 ; any pure state with $R>1$ is entangled. We'll see another use for the Schmidt decomposition below.

\section{Witness Operators}

An observable $\hat{W}$ is an entanglement witness if

$$
\langle\hat{W}\rangle=\operatorname{Tr}\left(\hat{W} \hat{\rho}_{\text {sep }}\right) \geq 0
$$

for all separable states $\hat{\rho}_{\text {sep }}$, and

$$
\langle\hat{W}\rangle=\operatorname{Tr}\left(\hat{W} \hat{\rho}_{e n t}\right)<0
$$

for at least one entangled state $\hat{\rho}_{\text {ent }} \cdot{ }^{3,5,6}$ Here $\operatorname{Tr}()$ refers to the trace of an operator. This means that if one measures $\langle\hat{W}\rangle<0$, one knows that the state $\hat{\rho}$ is entangled.

There are different ways to construct witness operators. The technique that we use is to note that if our experimentally produced state is "close enough" (in Hilbert space) to a particular entangled pure state $\left|\psi_{\text {ent }}\right\rangle$, it will be entangled as well. As such we construct the witness operator ${ }^{6}$

$$
\hat{W}=\alpha \hat{1}-\hat{\rho}_{e n t}=\alpha \hat{1}-\left|\psi_{e n t}\right\rangle\left\langle\psi_{e n t}\right|
$$

In order to ensure that this operator meets the definition of an entanglement witness, the constant $\alpha$ is chosen to have the minimum value possible such that $\hat{W}$ satisfies Eq. (5):

$$
\langle\hat{W}\rangle=\alpha\langle\hat{1}\rangle-\operatorname{Tr}\left(\left|\psi_{\text {ent }}\right\rangle\left\langle\psi_{\text {ent }}\right| \hat{\rho}_{\text {sep }}\right) \geq 0
$$

We thus require $\alpha$ to be given by

$$
\alpha=\max \operatorname{Tr}\left(\left|\psi_{\text {ent }}\right\rangle\left\langle\psi_{\text {ent }}\right| \hat{\rho}_{\text {sep }}\right) \text {. }
$$

Actually performing the maximization in Eq. (9) is beyond the scope of this article. It can be shown that $\alpha$ is given by the square of the maximum Schmidt coefficient of $\left|\psi_{\text {ent }}\right\rangle, \lambda_{i \max } \cdot{ }^{6,13}$

The two entangled states we are interested in detecting are the Bell states of two photons

$$
\left|\Phi^{ \pm}\right\rangle=\frac{1}{\sqrt{2}}(|H H\rangle \pm|V V\rangle)
$$

where $H$ and $V$ correspond to horizontally and vertically polarized photons. This is the Schmidt decomposition of $\left|\Phi^{ \pm}\right\rangle$, so the maximum Schmidt coefficient is $1 / \sqrt{2}$, and the witness operators are

$$
\begin{aligned}
\hat{W}^{ \pm}= & \frac{1}{2} \hat{1}-\left|\Phi^{ \pm}\right\rangle\left\langle\Phi^{ \pm}\right| \\
= & \frac{1}{2}[\hat{1}-|H H\rangle\langle H H|-| V V\rangle\langle V V| \\
& \mp(|H H\rangle\langle V V|+| V V\rangle\langle H H|)] .
\end{aligned}
$$

In the laboratory, we are able to perform local, projective measurements. That is, both Alice and Bob perform projective measurements on their respective particles. The first two terms after the $\hat{1}$ in Eq. (11) take this form, but the two terms in parentheses don't. 
However, we recognize that Alice and Bob are not limited to performing measurements in the horizontalvertical basis. Define the diagonal and antidiagonal $\left( \pm 45^{\circ}\right.$ linear $)$, and the left- and right- circular polarization states as

$$
\begin{gathered}
|D\rangle=\frac{1}{\sqrt{2}}(|H\rangle+|V\rangle),|A\rangle=\frac{1}{\sqrt{2}}(|H\rangle-|V\rangle) \\
|L\rangle=\frac{1}{\sqrt{2}}(|H\rangle+i|V\rangle),|R\rangle=\frac{1}{\sqrt{2}}(|H\rangle-i|V\rangle) .
\end{gathered}
$$

We rewrite our witness using these operators as:

$$
\begin{aligned}
\hat{W}^{ \pm}= & \frac{1}{2}[\hat{1}-|H H\rangle\langle H H|-| V V\rangle\langle V V| \mp(|D D\rangle\langle D D| \\
& +|A A\rangle\langle A A|-| L L\rangle\langle L L|-| R R\rangle\langle R R|)] .
\end{aligned}
$$

Defining $P(a, b)$ to be the joint probability that Alice measures her photon to have polarization $a$, and Bob measures his photon to have polarization $b$, we find that the expectation value of the witness operators is

$$
\begin{aligned}
\left\langle\hat{W}^{ \pm}\right\rangle= & \frac{1}{2}\{1-P(H, H)-P(V, V) \mp[P(D, D) \\
& +P(A, A)-P(L, L)-P(R, R)]\} .
\end{aligned}
$$

\section{EXPERIMENTS}

Our experiments are similar to those performed in Ref. [7], but we use equipment that is currently found in many undergraduate laboratories. ${ }^{4}$ The experimental apparatus is shown in Fig. 1. A $405 \mathrm{~nm}$ laser diode pumps a pair of Type-I beta-barium borate crystals, whose axes are oriented at right angles with respect to each other. Down converted photons pass through a series of wave plates and polarizing beam splitters, before being focused onto multimode optical fibers and detected with single-photon counting modules.

The polarization states of the down converted photon pairs are adjusted using the techniques of previous experiments. ${ }^{4,8}$ The states that we are trying to produce take the form

$$
|\Phi(\phi)\rangle=\frac{1}{\sqrt{2}}\left(|H H\rangle+e^{i \phi}|V V\rangle\right)
$$

The birefringent plate in the pump beam is mounted on a tilt stage with a micrometer, and is used to adjust the relative phase $\phi$; note that $\phi=0$ yields $\left|\Phi^{+}\right\rangle$and $\phi=\pi$ yields $\left|\Phi^{-}\right\rangle$. The techniques described in Refs.

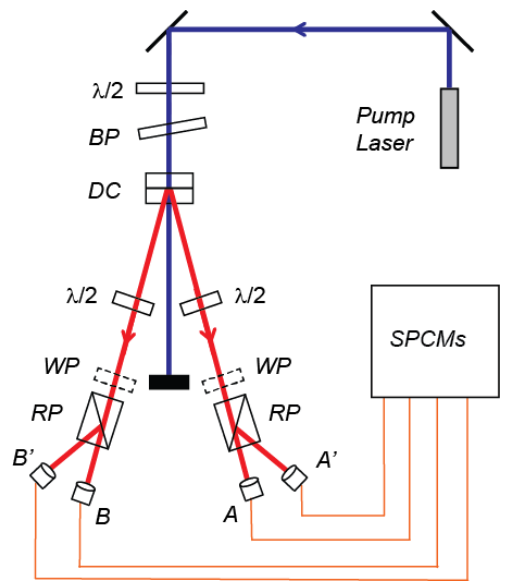

FIGURE 1. The experimental apparatus. Here $\lambda / 2$ denotes a half-wave plate, $B P$ denotes a birefringent plate, $D C$ denotes down conversion crystals, $W P$ denotes the wave plates used to do the measurement projections, $R P$ denotes a Rochon polarizer, and SPCMs are the single-photon counting modules.

4 and 8 allow us to easily determine $\phi=0$ and $\phi=\pi$. We extrapolate between these two tilt angles to determine the phase angle of the state.

However, our experimentally produced states are not pure. We model our states as

$$
\begin{aligned}
\hat{\rho} & =p|\Phi(\phi)\rangle\langle\Phi(\phi)| \\
& +\frac{1-p}{2}(|H H\rangle\langle H H|+| V V\rangle\langle V V|) .
\end{aligned}
$$

This density operator represents our photons as being in the entangled state $|\Phi(\phi)\rangle$ with probability $p$, and in an equal mixture of the states $|H H\rangle$ and $|V V\rangle$ with probability $1-p$.

With the optional wave plates removed (see Fig. 1) horizontally polarized photon pairs are directed to detectors $A$ and $B$, and vertically polarized photons are directed to detectors $A^{\prime}$ and $B^{\prime}$. We can thus measure the probabilities $P(H, H)$ and $P(V, V)$. The probabilities of detecting diagonal and antidiagonal photon pairs are obtained by inserting properly oriented half-wave plates before the Rochon polarizers. To measure the circular polarization probabilities we insert properly oriented quarter-wave plates.

Figure 2 shows the experimental data for our two witnesses, and the CHSH parameter $S .{ }^{8,9}$ In Fig. 2(a) we see that when we are creating $\left|\Phi^{+}\right\rangle$( $\phi$ near 0$)$, $\left\langle\hat{W}^{+}\right\rangle$indicates that the state is entangled, and $\left\langle\hat{W}^{-}\right\rangle$ does not. This is as we would expect, because $\hat{W}^{+}$is 


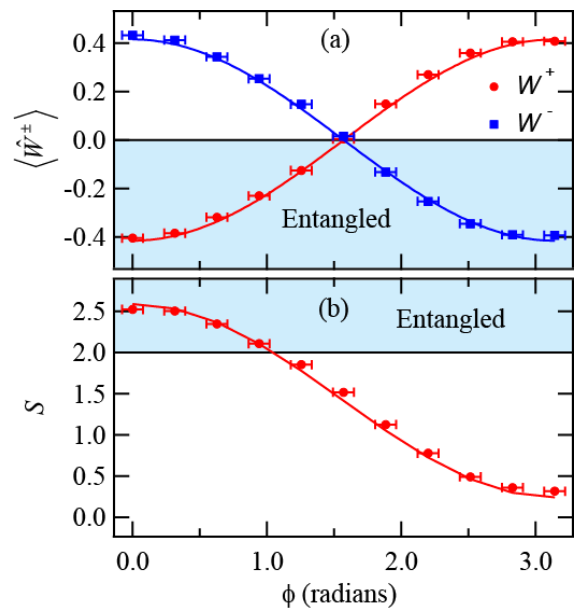

FIGURE 2. (a) $\left\langle\hat{W}^{+}\right\rangle$(red circles) and $\left\langle\hat{W}^{-}\right\rangle$(blue squares) are plotted as a function of the entangled state phase, $\phi$. (b) The CHSH parameter $S$ is plotted as a function of the entangled state phase, $\phi$. The points are experimental data, while the solid lines are theoretical predictions. Statistical (vertical) error bars are smaller than the markers. Horizontal error bars are $\pm \pi / 40$, which is our best estimate of how accurately we can set the phases.

constructed to witness this entangled state, while $\hat{W}^{-}$ is not. Their behavior switches as $\phi$ approaches $\pi$ and we are constructing state $\left|\Phi^{-}\right\rangle$. Note that the version of the CHSH inequality that we use detects entanglement in $\left|\Phi^{+}\right\rangle$when $S>2$. However, $\hat{W}^{+}$ does a "better" job of detecting this entanglement: $\left\langle\hat{W}^{+}\right\rangle$indicates that the point at $\phi \cong 1.25 \mathrm{rad}$ is entangled, while $S$ does not.

The expectation values $\left\langle\hat{W}^{ \pm}\right\rangle$are obtained from the same data, but computed differently. The data for $S$ is obtained separately because it requires different measurement settings. Our technique for obtaining the measurements in Fig. 2 is to set the value of $\phi$, measure $\left\langle\hat{W}^{ \pm}\right\rangle$and $S$ one after the other, then change $\phi$ and repeat. We note that at $\phi=0$ in Fig. 2 $\left\langle\hat{W}^{+}\right\rangle=-0.4042 \pm 0.0025$, which indicates that the state is entangled by over 160 standard deviations for $300 \mathrm{~s}$ of counting time. This same state yields $S=2.521 \pm 0.012$, which violates the classical inequality by 40 standard deviations for $400 \mathrm{~s}$ of counting time.

For states described by Eq. (17), the theoretical expectation values of the witness operators, are

$$
\left\langle\hat{W}^{ \pm}\right\rangle=\mp \frac{p}{2} \cos \phi
$$

We treat $p$ as a free parameter, and use it to fit our data for $\left\langle\hat{W}^{+}\right\rangle$; we find that $p=0.83 \pm 0.01$. Once this value has been determined for $\left\langle\hat{W}^{+}\right\rangle$, we use it to determine the theoretical predictions for $\left\langle\hat{W}^{-}\right\rangle$and $S$. Thus, we use one free parameter for all three theoretical curves shown in Fig. 2.

\section{CONCLUSIONS}

We have experimentally measured the expectation values of two different entanglement witness operators $\left\langle\hat{W}^{ \pm}\right\rangle$in an undergraduate laboratory, and compared them to measurements of the CHSH parameter $S$. Determining $\left\langle\hat{W}^{ \pm}\right\rangle$is "easier" in that they require only three measurements, as compared to four measurements for $S$. The witness operators also indicate entanglement for states that $S$ does not, and they yield a larger violation of classical physics (in terms of the number of standard deviations that a classical inequality is violated).

\section{ACKNOWLEDGMENTS}

We acknowledge the support of Whitman College.

\section{REFERENCES}

1. M. A. Nielsen and I. L. Chuang, Quantum Computation and Quantum Information (Cambridge Univ. Press, Cambridge, 2000).

2. N. Gisin and A. Peres, Phys. Lett. A 162, 15-17 (1992).

3. B. M. Terhal, Theor. Comput. Sci. 287, 313-335 (2002).

4. M. Beck, Quantum Mechanics: Theory and Experiment (Oxford Univ. Press, Oxford, 2012).

5. Michał Horodecki, Paweł Horodecki, and Ryszard Horodecki, Phys. Lett. A 223, 1-8 (1996).

6. O. Gühne and G. Toth, Phys. Rep. 474, 1-75 (2009).

7. M. Barbieri et al., Phys. Rev. Lett. 91, 4 (2003).

8. D. Dehlinger and M. W. Mitchell, Am. J. Phys. 70, 898902 (2002).

9. D. Dehlinger and M. W. Mitchell, Am. J. Phys. 70, 903910 (2002).

10. J. A. Carlson, M. D. Olmstead, and M. Beck, Am. J. Phys. 74, 180-186 (2006).

11. E. J. Galvez, Am. J. Phys. 78, 510-519 (2010).

12. E. Dederick and M. Beck, Am. J. Phys. 82, 962-971 (2014).

13. Mohamed Bourennane et al., Phys. Rev. Lett. 92, 087902 (2004) 